\title{
Molecular Mapping and Characterization of QBp.caas-3BL for Black Point Resistance in Wheat (Triticum Aestivum L.)
}

Cuihe Liu

Chinese Academy of Agricultural Sciences Institute of Crop Sciences Jie Song

Chinese Academy of Agricultural Sciences Institute of Crop Sciences

Siyang Liu

Chinese Academy of Agricultural Sciences Cotton Research Institute

Jingdong Liu

Chinese Academy of Agricultural Sciences Institute of Crop Sciences

\section{Dengan Xu}

Chinese Academy of Agricultural Sciences Institute of Crop Sciences

Xiuling Tian

Chinese Academy of Agricultural Sciences Institute of Crop Sciences

Yingjie Bian

Chinese Academy of Agricultural Sciences Institute of Crop Sciences

Yachao Dong

Chinese Academy of Agricultural Sciences Institute of Crop Sciences

Fengju Wang

Chinese Academy of Agricultural Sciences Institute of Crop Sciences

Rongge Wang

Farm of Seed Production of Gaoyi, Hebei

Zhonghu He

Chinese Academy of Agricultural Sciences Institute of Crop Sciences

Xianchun Xia

Chinese Academy of Agricultural Sciences Institute of Crop Sciences

SHUANGHE CAO ( $\square$ CAOSHUANGHE@CAAS.CN)

Chinese Academy of Agricultural Sciences Institute of Crop Sciences https://orcid.org/0000-00022905-0728

\section{Research Article}

Keywords: Candidate gene prediction, Fine mapping, Kernel smudge, Marker-assisted selection, Wheat 
Posted Date: March 30th, 2021

DOI: https://doi.org/10.21203/rs.3.rs-323020/v1

License: (c) (i) This work is licensed under a Creative Commons Attribution 4.0 International License. Read Full License 


\section{Abstract}

Wheat black point, which occurs in most wheat growing regions of the world, is detrimental to grain appearance, processing and nutrient quality. Mining and characterization of genetic loci for black point resistance is helpful for breeding resistant wheat cultivars. We previously identified a major QTL QBp.caas-3BL in a recombinant inbred line (RIL) population of Linmai 2/Zhong 892 across five environments. Here we confirmed the QTL in two additional environments. The genetic region of QBp.caas-3BL was enriched with newly developed markers. Using four sets of near isogenic lines QBp.caas-3BL was narrowed down to a physical interval of approximately $1.7 \mathrm{Mb}$, including five annotated genes according to IWGSC reference genome. TraesCS3B02G404300, TraesCS3B02G404600 and TraesCS3B02G404700 were predicted as candidate genes based on the analyses of sequence polymorphisms and differential expression. We also converted a SNP of TraesCS3B02G404700 into a breeding-applicable KASP marker and verified its efficacy for marker-assisted breeding in a panel of germplasm. The findings not only lay a foundation for map-based cloning of QBp.caas-3BL but also provide a useful marker for selection of resistant cultivars genotypes in wheat breeding.

\section{Key Message}

We fine-mapped $Q B$.caas-3BL for black point resistance in an interval of $1.7 \mathrm{Mb}$ containing five highconfidence annotated genes and developed a KASP marker suitable for selection of QBp.caas-3BL.

\section{Introduction}

Wheat is an important staple crop that provides about $20 \%$ of calories for humankind. Black point (also known as kernel smudge) is a serious disease characterized by dark discoloration at the embryo end of wheat grains and occurs in most wheat-growing regions of the world. It causes inferior grain appearance, processing and nutrient quality as well as lower seed vigor (Liu et al. 2016). Mining and characterization of genetic loci for black point resistance is helpful for breeding cultivars with resistance to the disease.

To date, relatively few genetic studies have been performed on black point response in wheat. A total of nine QTL for black point resistance were mapped on chromosomes 1D, 2A, 2B, 2D, 3D, 4A, 5A and 7A (2 QTL) in bi-parent mapping populations derived from crosses Cascades/AUS1408 and Sunco/Tasman, each explaining $4.0-18.4 \%$ of the phenotypic variance (Lehmensiek et al. 2004). Twenty-five loci associated with black point response on chromosomes 2A, 2B, 3A, 3B (2), 3D, 4B (2), 5A (3), 5B (3), 6A, 6B, 6D, 7A (5), 7B and 7D (2) explained phenotypic variances ranging from 7.9 to 18.0\% (Liu et al. 2017). Recently, Li et al. (2020) detected 23 loci associated with black point response on chromosomes 1B, 1D, 2B (2), 2D, 3A (2), 3B (2), 3D, 4A, 5A (2), 5B (2), 6B (3), 6D, 7A (2) and 7D (2), respectively. Lv et al. (2020) identified 386 marker-trait associations (MTAs) for black point response by genome-wide association analysis and three of the MTAs were further verified in a bi-parental population. These studies laid the foundation for map-based cloning of genes for resistance to black point, but fine mapping of the genetic loci has not been conducted thus far. 
In our previous study, nine QTL for black point resistance were mapped on chromosomes $2 A L, 2 B L, 3 A L$, $3 B L, 5 A S, 6 A, 7 A L(2)$ and 7BS in a recombinant inbred line (RIL) population derived from the Linmai 2/Zhong 892 cross (Liu et al. 2016). QBp.caas-3BL was detected across five environments and explained 6.0-12.2\% of the phenotypic variances, suggesting that it was a stable major QTL for black point resistance. Here $Q B$. caas-3BL was confirmed in another two environments. We created a series of near isogenic lines to fine-map QBp.caas-3BL and predicted its candidate genes. A cost-effective KASP marker was also developed to improve black point resistance in wheat breeding.

\section{Materials And Methods}

\section{Plant materials}

To confirm QBp.caas-3BL, we planted the Linmai 2/Zhong 892 RIL population in Anyang (Henan province) and Suixi (Anhui province) during the 2018-2019 cropping season. The residual heterozygous recombinant plants for $Q B$ p.caas-3BL were screened from the RIL population. Four sets of near isogenic lines (NILs) for QBp.caas-3BL were identified from the residual heterozygous recombinant plants and used for fine mapping (Table S1). The NILs were grown in single row plots of $2 \mathrm{~m}$ with approximately 40 plants per row and $20 \mathrm{~cm}$ between rows at Anyang during the 2018-2019 cropping season and Xinxiang (Henan province) during the 2019-2020 cropping season. A panel of 166 wheat cultivars from the HuangHuai Wheat Region was used to validate the genetic effect of $Q B$.caas-3BL on black point response (Liu et al. 2017).

\section{Phenotypic evaluation for black point}

Black point in mature grains was assessed manually at least 15 days after harvest. Each RIL or NIL was scored for black point response (obvious discoloration around the embryos) by counting the number of black point-affected grains among three samples of 200. The percentage of black point-affected kernels was taken as the black point score for each plot. To ensure the accuracy of phenotyping, black point of all materials was scored by the same person under similar illumination conditions.

\section{PCR-based genotyping by sequencing}

To enrich the target region of $Q B p$.caas-3BL, annotated genes were identified in the physical interval according to the International Wheat Genome Sequencing Consortium (IWGSC) RefSeq v1.1 (http://plants.ensembl.org/index.html). The sequences from some annotated genes were retrieved and aligned to design gene-specific primers by DNAMAN software. The polymorphic sites between parents Linmai 2 and Zhong 892 were used to develop markers for fine mapping of QBp.caas-3BL. PCR was performed in reaction mixtures of $15 \mu$ containing $7.5 \mu$ of $2 \times$ Taq PCR Mix (Beijing HT-biotech Co., Ltd., Beijing), 5 pmol of each primer and 50-100 ng of genomic DNA using an ABI Applied Biosystems Veriti 96 Well Thermal Cycler (Gene Co., Ltd., Shanghai). PCR amplification was performed at $95^{\circ} \mathrm{C}$ for 3 min, followed by 35 cycles $\left(94^{\circ} \mathrm{C}\right.$ for $30 \mathrm{~s}, 59-61^{\circ} \mathrm{C}$ for $30 \mathrm{~s}$ and $72^{\circ} \mathrm{C}$ for $1 \mathrm{~min}$ ) and a final extension at $72^{\circ} \mathrm{C}$ for 7 min. Primer pairs and their annealing temperature for PCR were listed in Table S2. PCR products 
were recovered from 1.5\% agarose gels and purified by TIANgel MIDI Purification Kit (Tiangen, Biotechnology Co., Ltd., Beijing). The sequencing of target PCR products was performed by BGI Genomics Co., Ltd in Beijing.

\section{Development of a KASP marker}

PolyMarker software (http://polymarker.tgac.ac.uk/) was used to design KASP markers for target polymorphic sites (Table S3). The PCR cycling conditions were $94^{\circ} \mathrm{C}$ for $15 \mathrm{~min}$, followed by 10 touchdown cycles $\left(94^{\circ} \mathrm{C}\right.$ for $20 \mathrm{~s} ; 62^{\circ} \mathrm{C}$ to $55^{\circ} \mathrm{C}$ for $\left.60 \mathrm{~s}\right), 26$ cycles of $94^{\circ} \mathrm{C}$ for $20 \mathrm{~s}$ and $55^{\circ} \mathrm{C}$ for $60 \mathrm{~s}, 6$ cycles of $94^{\circ} \mathrm{C}$ for $20 \mathrm{~s}$ and $57^{\circ} \mathrm{C}$ for $60 \mathrm{~s}$, and one final acquisition cycle at $16^{\circ} \mathrm{C}$ for $60 \mathrm{~s}$ (Collins et al. 2018). The KASP platform of LGC Co., Ltd was used to carry out genotyping and statistical analyses.

\section{Quantitative PCR}

Grains of Linmai2 and Zhong892 were sampled 25 days after flowering and used to isolate total RNA by a Total RNA Extraction Kit (Beijing TransGen Biotechnology, Cat\#ER301-01). cDNA was generated with a PrimeScript RT Reagent Kit plus gDNA Eraser (TaKaRa, Dalian). Quantitative PCR (qPCR) was performed on cDNA samples produced from three biological replicates in a BioRad CFX system using iTaq Universal SYBR Green Supermix (BioRad, Beijing). The primer pairs for gene-specific qPCR were designed according to the gene annotation from RefSeq v1.1 (Table S4). The profile of qPCR was as follows: $95^{\circ} \mathrm{C}$ for 2 min, followed by 40 cycles of $95^{\circ} \mathrm{C}$ for $5 \mathrm{~s}$ and $59^{\circ} \mathrm{C}$ for $20 \mathrm{~s}$. A final dissociation stage was run to generate a melting curve for judgement of amplification specificity. Relative gene expression was normalized to elongation factor 1 alpha (EF1a) using the $2^{-\Delta \Delta C t}$ equation.

\section{Linkage mapping and QTL analysis}

A genetic linkage map for QBp.caas-3BL was constructed using JoinMap 4.0 (http://www.kyazma.com). QTL mapping was conducted by inclusive composite interval mapping (ICIM) in IciMapping 4.0 software (https://www.isbreeding.net) with a LOD threshold of 3.0. The means of phenotypic data for RILs in each environment were used for QTL analysis.

\section{Statistical analysis}

Association analysis for declaration of significance at $P<0.05$ in KASP marker validation was conducted by student's $t$-tests in Excel. The Excel software was also used to calculate the means, standard deviation and coefficients of variation of phenotypic data.

\section{Results}

\section{Confirmation of QBp.caas-3BL}

In the previous study, QBp.caas-3BL was identified as a major QTL for black point in the RIL population of Linmai 2/Zhong 892 (Liu et al. 2016). To confirm the presence of QBp.caas-3BL, we re-investigated black 
point response of the RIL population in two additional environments (2019 Anyang and 2019 Suixi). Based on the phenotypes in these two environments, the QTL QBp.caas-3BL was confirmed with a consistent genetic location across environments (Fig. 1A), explaining 6.6-12.4\% of the phenotypic variance. These results confirmed that $Q B$ p.caas-3BL is a stable QTL for black point.

\section{Generation of secondary mapping populations for QBp.caas-3BL}

To narrow down the target interval of $Q B p$.caas-3BL, we created secondary mapping populations. Based on the genotyping data by the wheat $90 \mathrm{~K}$ SNP chip, four RILs with residual heterozygosity in the QBp.caas-3BL target region were identified using markers. We genotyped approximately 2000 individual plants from the heterozygous recombinant lines using the flanking markers RAC875_rep_c105184_88 (M1) and Tdurum_contig67350_494 (M12) (Fig. 1A; Table S2). Each of the so-called residual heterozygous recombinant lines had homozygous plants with contrasting genotypes in the target region of $Q B$. caas-3BL. Since the plants from each residual heterozygous recombinant line harbored contrasting genotypes in the target region but were homozygous for most other regions of the genome based on SNP arrays, they were treated as NILs of QBp.caas-3BL. Four sets of QBp.caas-3BL NILs were selected and used to create secondary populations for fine mapping (Table S1).

Fine mapping of QBp.caas-3BL

To enrich the interval of QBp.caas-3BL, we identified polymorphic sites of six genes within the target region based on the IWGSC RefSeq v1.1 (http://plants.ensembl.org/index.html) and developed genespecific markers (Table S2). Six chip-based SNPs that were identified previously as flanking markers of QBp.caas-3BL were also converted into sequencing-based markers. Using these markers, we mapped QBp.caas-3BL to a smaller genetic region (Fig. 1A). QBp.caas-3BL was further delimited in a physical interval between M8 (TraesCS3B02G404200) and M10 (TraesCS3B02G404800) based on genotypic and phenotypic analyses of the secondary mapping populations (Fig. 1B; Table S1). The physical interval spanned 1.7 Mb, including five high-confidence annotated genes, TraesCS3B02G404300, TraesCS3B02G404400, TraesCS3B02G404500, TraesCS3B02G404600 and TraesCS3B01G404700 according to RefSeq v1.1 (Fig. 1C).

\section{Prediction Of Candidate Genes}

Among the five candidate genes, TraesCS3B02G404300, TraesCS3B02G404400, TraesCS3B02G404500 and TraesCS3B02G404600 are bZIP family transcription factors that are orthologous to ABSCISIC ACIDINSENSITIVE 5 (ABI5) (Fig. 1C and S1), whereas TraesCS3B02G404700 is a homolog of protein phosphatase (Fig. S2). To determine the candidate gene(s) in QBp.caas-3BL we compared the open reading frames (ORFs) of the five genes from parents Linmai 2 and Zhong 892 by sequencing. The ORFs of TraesCS3B02G404600 and TraesCS3B02G404700 contained sites polymorphic between Linmai 2 and Zhong 892. The SNP in TraesCS3B02G404600 was a synonymous mutation, whereas that of TraesCS3B02G404700 was a nonsynonymous mutation causing a serine-to-alanine substitution (Fig. 2A 
and 2B). We also investigated the expression patterns of the five genes in Wheat Expression Browser (http://wheat-expression.com/) and found that all were expressed in grains, suggesting that they could be involved in black point response (Fig. S3-7). All four ABI5 homologs, TraesCS3B02G404300,

TraesCS3B02G404400, TraesCS3B02G404500 and TraesCS3B02G404600, have spike- and seed-specific expression patterns, whereas TraesCS3B02G404700 is highly expressed in all tissues (Fig. S3-7). We further compared the transcriptional levels of the five genes in developing grains using QPCR assays and observed that TraesCS3B02G404300, TraesCS3B02G404600 and TraesCS3B02G404700 were differentially expressed between Linmai 2 and Zhong 892 (Fig. 2C). All three differentially expressed genes were down-regulated in Linmai 2 compared to Zhong 892 (Fig. 2C). Thus, each of TraesCS3B02G404300, TraesCS3B02G404600 and TraesCS3B02G404700 could be the causal gene of QBp.caas-3BL based on sequence polymorphism and differential expression.

\section{Development and validation of a KASP marker for QBp.caas-3BL}

TraesCS3B02G404700 was identified as a candidate gene for QBp.caas-3BL, with a SNP in its ORF between the parents. To develop a breeding-applicable marker for $Q B p$.caas-3BL, we converted the SNP into a cost-effective, high-throughput KASP marker (Fig. 2D; Table S3) and validated it in a panel of 166 wheat cultivars from the Huang-Huai Wheat Region, the largest wheat area in China. Association analysis indicated that sequence variation in TraesCS3B02G404700 was significantly associated with black point response (Table 1 ; Table S5).

Table 1

Genetic effects of QBp.caas-3BL on black point response in a panel of 166 wheat cultivars tested by the KASP marker developed from TraesCS3B02G404700

\begin{tabular}{|c|c|c|c|c|}
\hline Environment & Genotype & Number of accessions & Mean \pm SD (\%) & $P$-value \\
\hline \multirow[t]{2}{*}{ 2013AY } & 0 & 93 & $30.5 \pm 1.52$ & \multirow[t]{2}{*}{$0.0002^{\star \star}$} \\
\hline & 2 & 73 & $19.5 \pm 0.53$ & \\
\hline \multirow[t]{2}{*}{ 2014AY } & 0 & 93 & $20.6 \pm 2.15$ & \multirow[t]{2}{*}{$0.046^{*}$} \\
\hline & 2 & 73 & $16.4 \pm 0.74$ & \\
\hline \multirow[t]{2}{*}{$2013 S X$} & 0 & 93 & $29.3 \pm 0.75$ & \multirow[t]{2}{*}{$0.0003^{* *}$} \\
\hline & 2 & 73 & $19.8 \pm 0.56$ & \\
\hline \multicolumn{5}{|c|}{0,2 indicate the genotypes of Linhan 2 and Zhong 892, respectively. } \\
\hline \multicolumn{5}{|c|}{$*$ and $* *$ indicate significant differences at $P<0.05$ and $P<0.01$, respectively. } \\
\hline \multicolumn{5}{|c|}{$\begin{array}{l}2013 \mathrm{AY}, 2014 \mathrm{AY} \text { and } 2013 S \mathrm{~S} \text { represent different cropping seasons in Anyang (AY) and Suixi (SX), } \\
\text { respectively. }\end{array}$} \\
\hline
\end{tabular}

\section{Discussion}


In the present study we confirmed the effect of $Q B p . c a a s-3 B L$ in additional two environments, in agreement with Liu et al. (2016). QBp.caas-3BL was further mapped into a physical interval of approximately $1.7 \mathrm{Mb}$ from which candidate genes were also identified. These results provide important reference information for map-based cloning and marker-assisted selection of QBp.caas-3BL. Based on a polymorphic site in TraesCS3B02G404700, a candidate gene for QBp.caas-3BL, we developed a costeffective and high-throughput KASP marker. Association analysis showed that QBp.caas-3BL had a significant effect on black point resistance using the KASP marker in a diverse panel of 166 wheat cultivars, suggesting that the marker is a breeding-applicable tool for marker-assisted selection of black point resistance. The additional field data obtained in the study confirmed that $Q B p$.caas-3BL is a stable major QTL for black point response and could be useful to improve black point resistance in wheat breeding.

\section{Functional prediction of candidate genes for QBp.caas-3BL}

The cause of black point is complicated; the symptom have been attributed to fungal infection, enzymatic browning and environmental stress, such as high humidity and temperature (March et al. 2007; Fernandez and Conner 2011; Busman et al. 2012; Fernandez et al. 2014). In this study we mapped QBp.caas-3BL to a physical interval of $1.7 \mathrm{Mb}$, and determined TraesCS3B02G404300,

TraesCS3B02G404600 and TraesCS3B02G404700 as candidate genes. Phylogenetic analysis showed that TraesCS3B02G404300 and TraesCS3B02G404600 are probably resulted from tandem duplication events (Fig. S1A). Additionally, it appears that the tandem duplication events have happened in Triticum dicoccoides (also known as wild emmer), the putative progenitor of wheat subgenomes $A$ and $B$, according to BLASTN against WEWSeq_v.1.0 (Genomic sequence) (http://plants.ensembl.org/Triticum_dicoccoides/Tools/Blast/Results?r=3B:639752637639756075; tl=7tHVuMIVeE1hFSOL-20388519). TraesCS3B02G404300 and TraesCS3B02G404600 are orthologous to $A B I 5$ in Arabidopsis and OSABI5-1 in rice (Fig. S1A). $A B I 5$ has pleiotropic effects on plant growth and development and is also involved in response to biotic and abiotic stresses. In Arabidopsis, ABI5 (AT2G36270) is a key regulator in ABA signaling during seed maturation and germination and modulates late embryogenesis abundant genes during both developmental stages (Finkelstein et al. 2000; Ibarra et al. 2016). Bi et al. (2017) showed that AtABI5 played a role in regulating the homeostasis of reactive oxygen species (ROS) by activating CATALASE1 transcription during seed germination. OsABI5 (Os01g0859300) was reported to regulate fertility and stress tolerance in rice (Zou et al. 2008). TaABF1-3A (TraesCS3A02G371800), an ortholog of TraesCS3B02G404300, was shown to participate in grain development by physically interacting with $P K A B A 1$, a signaling component in the ABA-suppression of GA-induced gene expression in cereal grains (Fig. S1; Johnson et al. 2008). Harris et al. (2013) further validated that $T a A B F 1$ functioned as an integrator of $A B A$ and GA signaling in aleurone cells of grains. Recently, overexpression of TaABI5 (99.4\% similarity with TraesCS3D02G364900 in IWGSC RefSeq v1.1) in Arabidopsis transformants exhibited high sensitivity to ABA and repressed germination (Utsugi et al. 
2020). Hence, TraesCS3B02G404300 and TraesCS3B02G404600, homologs of $A B I 5$, are probably involved in seed development by modulating $A B A$ and $G A$ signaling.

TraesCS3B02G404700, the third candidate gene of QBp.caas-3BL, is orthologous to OsPP16 in rice, whereas an ortholog is not detected in Arabidopsis based on phylogenetic analyses (Fig. S2). OsPP16 belongs to a dual specificity phosphatase (DUSP) clade (Singh et al. 2010). As a member of the tyrosine phosphatase superfamily, dual specificity phosphatase (DUSP) can dephosphorylate tyrosine, serine and threonine residues, and is involved in many basic physiological activities such as plant growth, development and abiotic/biotic stress response (Jiang et al. 2018). OsPFA-DSP1, an atypical dualspecific phosphatase (PFA-DSP1) subfamily member in rice, may act as a negative regulator in drought stress responses (Liu et al. 2012). DUSP IBR5 in Arabidopsis is involved in regulation of R protein CHS3mediated temperature dependent responses (Liu et al. 2015). Thus, it appears that TraesCS3B02G404700 is involved in response to abiotic/biotic stress, a postulated cause of black point.

In summary, the functional prediction of candidate genes in $Q B P$.caas-3BL lays a strong foundation for identification of its causal gene and ascertainment of the underlying mechanism. Transgenic experiments are ongoing to validate the functions of the candidate genes.

\section{Declarations}

\section{Acknowledgments}

We thank Prof. R. A. Mclntosh, Plant Breeding Institute, University of Sydney, for reviewing this manuscript. This work was funded by the National Natural Science Foundation of China (31971929, 31961143007), the National Key Research and Development Program of China (2016YFD0101802, 2016YFE0108600), and CAAS Science and Technology Innovation Program.

\section{Conflict of interest:}

We declare no conflicts of interest in regard to this manuscript.

\section{Ethical Standards:}

These experiments complied with the ethical standards in China.

\section{References}

1. Bi C, Ma Y, Wu Z, Yu YT, Liang S, Lu K, Wang XF (2017) Arabidopsis ABI5 plays a role in regulating ROS homeostasis by activating CATALASE 1 transcription in seed germination. Plant Mol Biol 94:197-213

2. Busman M, Desjardins AE, Proctor RH (2012) Analysis of fumonisin contamination and the presence of Fusarium in wheat with kernel black point disease in the US. Food Addit Contam 29:1092-1100 
3. Collins D, Emebiri L, Tan MC, El Bouhssini M, Wildman O (2018) Association of KASP markers with Hessian fly resistance in wheat of diverse origin. Euphytica 214:144

4. Fernandez MR, Conner RL (2011) Black point and smudge in wheat. Prairie Soils Crops 4:158-164

5. Fernandez MR, Wang H, Singh AK (2014) Impact of seed discoloration on emergence and early plant growth of durum wheat at different soil gravimetric water contents. Can J Plant Pathol 36:509-516

6. Finkelstein RR, Lynch TJ (2000) The Arabidopsis abscisic acid response gene $A B I 5$ encodes a basic leucine zipper transcription factor. Plant Cell 12:599-609

7. Harris LJ, Martinez SA, Keyser BR, Dyer WE, Johnson RR (2013) Functional analysis of TaABF1 during abscisic acid and gibberellin signaling in aleurone cells of cereal grains. Seed Sci Res 23:8998

8. Ibarra SE, Tognacca RS, Dave A, Graham IA, Sánchez RA, Botto JF (2016) Molecular mechanisms underlying the entrance in secondary dormancy of Arabidopsis seeds. Plant Cell Environ 39:213-221

9. Jiang L, Chen Y, Luo L, Peck SC. (2018) Central roles and regulatory mechanisms of dual-specificity MAPK phosphatases in developmental and stress signaling. Front Plant Sci 9:1697

10. Johnson RR, Shin M, Shen JQ (2008) The wheat PKABA1-interacting factor TaABF1 mediates both abscisic acid-suppressed and abscisic acid-induced gene expression in bombarded aleurone cells. Plant Mol Biol 68:93-103

11. Lehmensiek A, Campbell AW, Williamson PM, Michalowitz M, Sutherland MW, Daggard GE (2004) QTLs for black-point resistance in wheat and the identification of potential markers for use in breeding programs. Plant Breed 123:410-416

12. Li Q, Niu H, Xu K, Xu Q, Wang S, Liang X, Jiang Y, Niu J (2020) GWAS for resistance against black point caused by Bipolaris sorokiniana in wheat. J Cereal Sci 91:e102859

13. Liu B, Fan JQ, Zhang Y, Mu PQ, Wang P, Su JB, Lai HH, Li SW, Feng DR, Wang JF, Wang HB (2012) OSPFA-DSP1, a rice protein tyrosine phosphatase, negatively regulates drought stress responses in transgenic tobacco and rice plants. Plant Cell Rep 31:1021-1032

14. Liu JY, Yang HB, Bao F, Ao K, Zhang XY, Zhang YL, Yang SH (2015) IBR5 modulates temperaturedependent, R protein CHS3-mediated defense responses in Arabidopsis. PLoS Genet 11:e1005584

15. Liu JD, He ZH, Wu L, Bai B, Wen WE, Xie CJ, Xia XC (2016) Genome-wide linkage mapping of QTL for black point reaction in bread wheat (Triticum aestivum L.). Theor Appl Genet 129:2179-2190

16. Liu JD, He ZH, Rasheed A, Wen WE, Yan J, Zhang PZ, Wan YX, Zhang Y, Xie CJ, Xia XC (2017) Genome-wide association mapping of black point reaction in common wheat (Triticum aestivum $\mathrm{L}$.). BMC Plant Biol 17:220-231

17. Lv GG, Dong ZD, Wang YD, Geng JY, Li J, Lv XL, Sun CW, Ren Y, Zhang JW, Chen F (2020) Identification of genetic loci of black point in Chinese common wheat by Genome-Wide Association Study and linkage mapping. Plant Dis 104:2005-2013

18. March TJ, Able JA, Schultz C, Able AJ (2007) A novel late embryo genesis abundant protein and peroxidase associated with black point in barley grains. Proteomics 7:3800-3808 
19. Singh A, Giri J, Kapoor S, Tyagi AK, Pandey GK (2010) Protein phosphatase complement in rice: genome-wide identification and transcriptional analysis under abiotic stress conditions and reproductive development. BMC Genomics 11:435-441

20. Utsugi S, Ashikawa I, Nakamura S, Shibasaka M. (2020) TaABI5, a wheat homolog of Arabidopsis thaliana ABA insensitive 5, controls seed germination. J Plant Res 133:245-256

21. Zou MJ, Guan YC, Ren HB, Zhang F, Chen F (2008) A bZIP transcription factor, OsABI5, is involved in rice fertility and stress tolerance. Plant Mol Biol 66:675-683

\section{Figures}

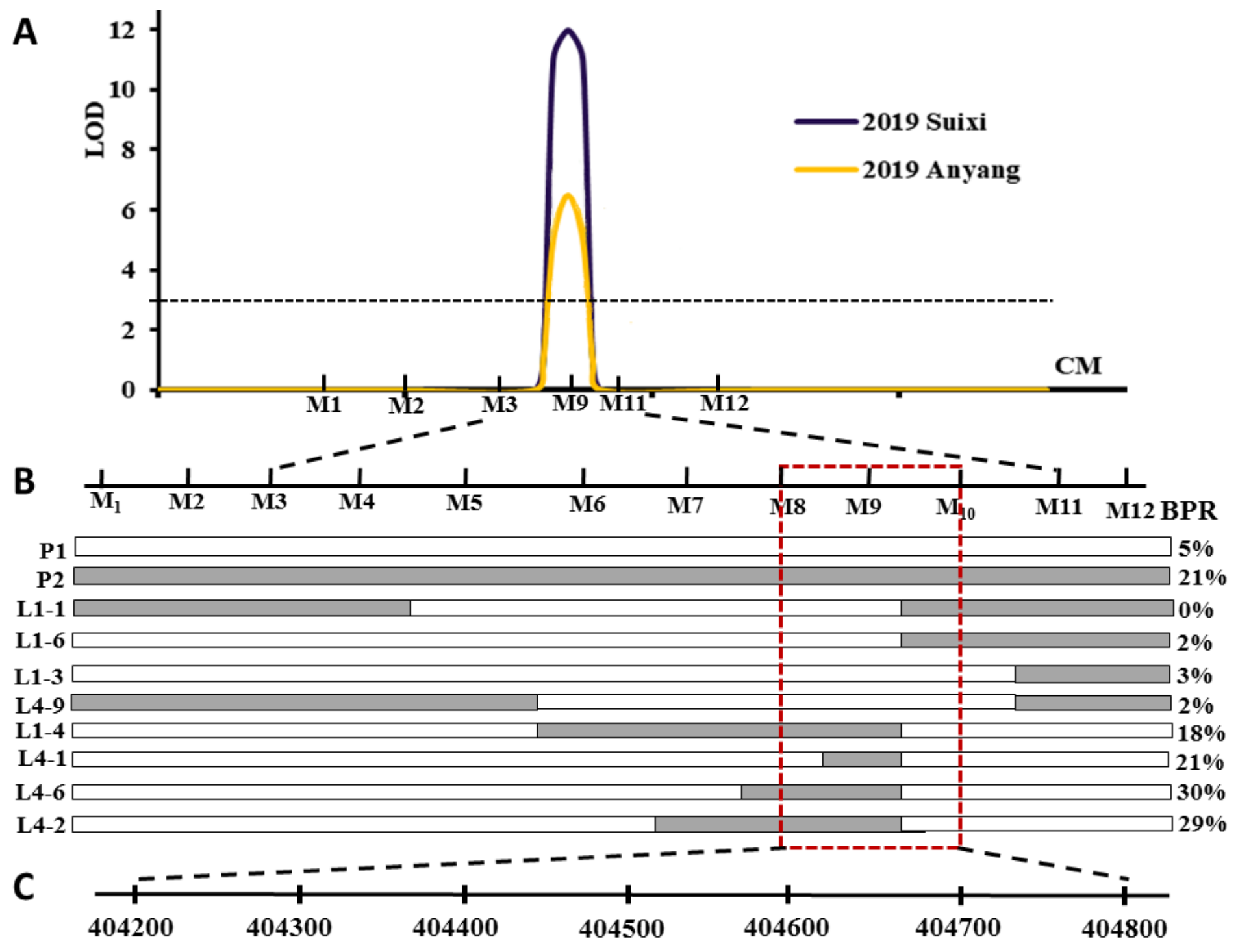

Figure 1

Genetic confirmation (A), fine mapping (B) and physical delimitation (C) of QBp.caas-3BL. M1-M12 are markers that correspond to Table S2; P1 and P2 represent Zhong 892 and Linmai 2, respectively; BPR, black point rate. 
A

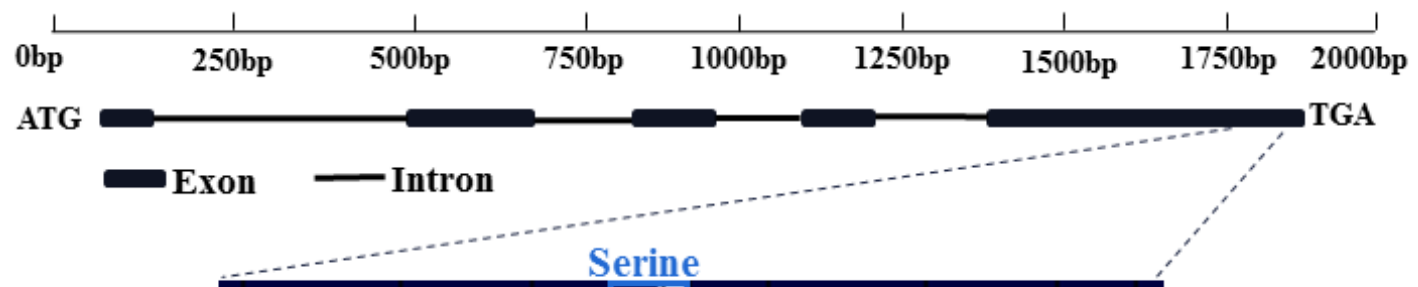

Linmai2 GACGAGTTGCAATACTOIGTGTGCGAGGCGGGCAC

Zhong892 GACGAGTTGCAATACTAGGTGTGCGAGGCGGGGCAC

Serine

B

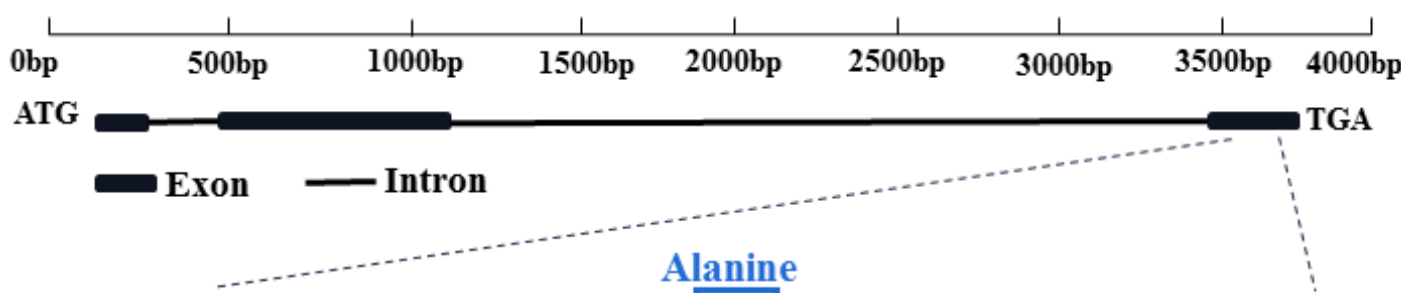

Linmai2 ATGGTCGAACACTTGCTT GCTCCCTCAAAAGGAAGTGGGAR

Zhong892 ATGGTCGAAACACTTGCTTIICTCCCTCAAAAGGAAGTGGGGAP

C
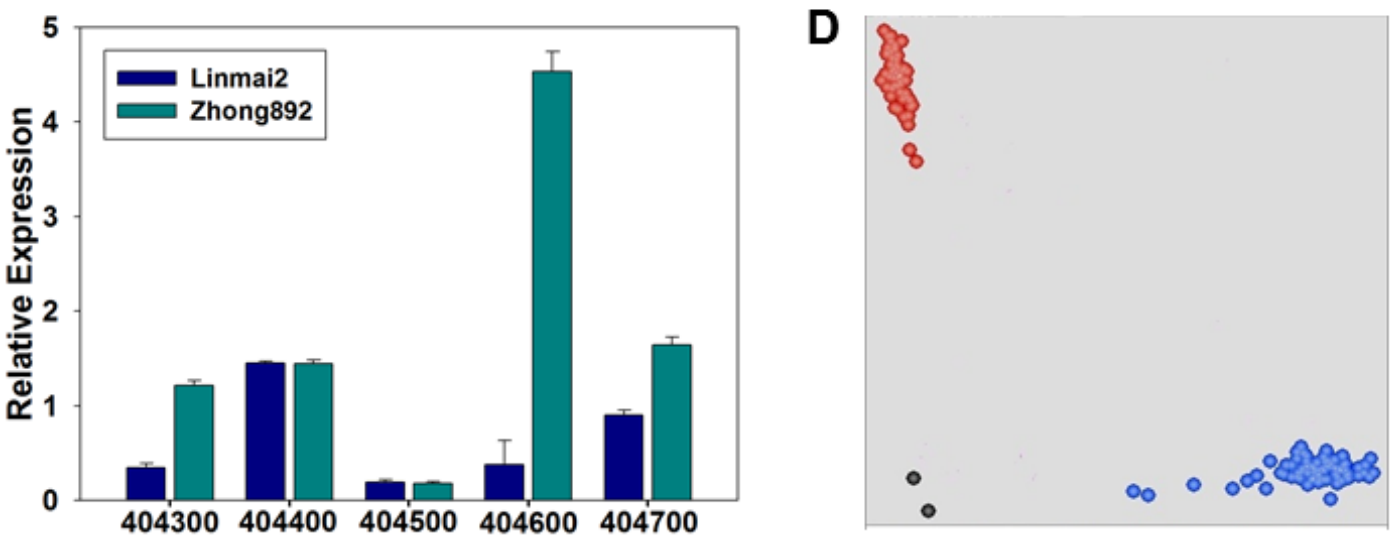

Figure 2

Prediction of candidate genes and development of a breeding marker for QBp.caas-3BL. Polymorphic sites between the parents in open reading frames of genes TraesCS3B02G404600 (A) and TraesCS3B02G404700 (B). (C) Differential expression of the annotated genes in the target region of QBp.caas-3BL in grains 25 days after flowering. (D) Genotyping of 166 wheat cultivars from the HuangHuai Wheat Region using the KASP marker developed from TraesCS3B02G404700.

\section{Supplementary Files}

This is a list of supplementary files associated with this preprint. Click to download.

- SupplementaryFigures.docx

- SupplementaryTables.xls 